\title{
Rare mutations in Pfmdr1 gene of Plasmodium falciparum detected in clinical isolates from patients treated with anti-malarial drug in Nigeria
}

\author{
Abel O. Idowu ${ }^{1,2}$, Wellington A. Oyibo ${ }^{4}$, Sanjib Bhattacharyya ${ }^{3}$, Manjeet Khubbar ${ }^{3}$, Udoma E. Mendie $^{2}$, \\ Violet V. Bumah ${ }^{6}$, Carolyn Black ${ }^{5}$, Joseph Igietseme $e^{5}$ and Anthony A. Azenabor ${ }^{1,2^{*}}$
}

\begin{abstract}
Background: Plasmodium falciparum, the deadliest causative agent of malaria, has high prevalence in Nigeria. Drug resistance causing failure of previously effective drugs has compromised anti-malarial treatment. On this basis, there is need for a proactive surveillance for resistance markers to the currently recommended artemisinin-based combination therapy (ACT), for early detection of resistance before it become widespread.

Methods: This study assessed anti-malarial resistance genes polymorphism in patients with uncomplicated $P$. falciparum malaria in Lagos, Nigeria. Sanger and Next Generation Sequencing (NGS) methods were used to screen for mutations in thirty-seven malaria positive blood samples targeting the $P$. falciparum chloroquine-resistance transporter (Pfcrt), P. falciparum multidrug-resistance 1 (Pfmdr1), and P. falciparum kelch 13 (Pfk13) genes, which have been previously associated with anti-malarial resistance.

Results: Expectedly, the NGS method was more proficient, detecting six Pfmdr1, seven Pfcrt and three Pfk 13 mutations in the studied clinical isolates from Nigeria, a malaria endemic area. These mutations included rare Pfmdr1 mutations, N504K, N649D, F938Y and S967N, which were previously unreported. In addition, there was moderate prevalence of the K76T mutation (34.6\%) associated with chloroquine and amodiaquine resistance, and high prevalence of the N86 wild type allele (92.3\%) associated with lumefantrine resistance.
\end{abstract}

Conclusion: Widespread circulation of mutations associated with resistance to current anti-malarial drugs could potentially limit effective malaria therapy in endemic populations.

Keywords: Malaria, Infectious disease epidemiology, Antimalarial gene polymorphism, Parasitic disease epidemiology and control, Plasmodium

\section{Background}

Plasmodium falciparum causes the highest number of malaria-related fatalities globally and has high prevalence in Nigeria [1, 2]. An estimated 212 million cases and 429,000 deaths due to malaria occurred in 2015,

\footnotetext{
*Correspondence: caxisonny@gmail.com

${ }^{1}$ Department of Biomedical Sciences, College of Health Sciences, University of Wisconsin, 2400 E. Hartford Avenue, Milwaukee, WI 53211, USA

Full list of author information is available at the end of the article
}

with $90 \%$ of the cases recorded in Africa [3]. Emergence and spread of mutations conferring drug resistance has limited the use of two affordable and previously effective anti-malarial drugs, chloroquine and sulfadoxine/ pyrimethamine, leading to the recommendation by the World Health Organization (WHO) of artemisininbased combination therapy (ACT), as the first-line treatment for malaria [4].

Artemisinin-based combinations are made up of a rapid but short-acting artemisinin and a long-acting partner drug combined to reduce the emergence of 
resistance. Artemether-lumefantrine (AL) and artesunate-amodiaquine are the recommended artemisininbased combinations for the treatment of uncomplicated malaria in Nigeria, and their suitability has been confirmed by results of recent therapeutic efficacy studies (TES), which showed a cure rate of greater than 95\% [5]. Deployment of ACT has led to a significant reduction in the number of malaria cases and fatalities [3], however, resistance to artemisinin, manifesting as delayed parasite clearance, has been observed in Western Cambodia since 2008 and has more recently been documented in extended areas of Southeast Asia [6-9]. The fear that artemisinin resistance could also spread through a similar trajectory as was observed with chloroquine and sulfadoxine/pyrimethamine resistance from Southeast Asia to Africa is now a real concern.

Apart from TES, which is a gold standard to monitor anti-malarial therapeutic efficacy, the WHO recommends the use of molecular markers to monitor the emergence of mutations associated with resistance to anti-malarial drugs as a proactive exercise to detect emerging resistance and prevent potential future treatment failure [10]. Several genetic markers associated with resistance to anti-malarial drugs have been identified. Mutations in the kelch propeller domain of the $k 13$ gene have been identified as molecular markers for artemisinin resistance by evaluating in vitro survival assays and observing delayed parasite clearance times and are currently being used to track the spread of resistance [7]. Currently, five validated (by in vivo and in vitro correlation data) and eight candidate (by correlation with delayed parasite clearance) $k 13$ mutations have been associated with artemisinin resistance [11]. Polymorphisms in the P. falciparum chloroquine resistance transporter gene ( $P f c r t)$ with substitutions at codon positions 72 to $76,97,220,271,326,356$, and 371 have been associated with in vivo and in vitro resistance to chloroquine and amodiaquine [12, 13]. The substitution at codon $76(\mathrm{~K} 76 \mathrm{~T})$ is the most predictive point mutation for chloroquine resistance [13].

The Pfmdr1 amino acid mutations that have been implicated in multidrug resistant phenotypes include the amino-terminal mutations (N86Y and Y184F) and the three carboxyl-terminal mutations (S1034C, N1042D and D1246Y) on the Pfmdr1 gene. These mutations have been reported to increase resistance to chloroquine, as well as modulate malaria parasite sensitivities to multiple drugs such as mefloquine, amodiaquine, quinine and halofantrine [14-16]. Mutations in Pfmdr1 is further suspected to be involved in parasite susceptibility to each of the ACT partner drugs as well as trophozoite-stage parasite sensitivity to artemisinin derivatives [17-19].
Recent studies have evaluated the emergence of resistance to piperaquine, an artemisinin partner drug $[20,21]$, with one study confirming that 14 out of 40 clinical isolates exhibited piperaquine resistance, leading to the recommendation for a change of treatment in Vietnam [22]. To date, no molecular marker for piperaquine resistance has been identified [10], although a recent study has linked a surrogate marker to piperaquine resistance [23], highlighting the need for the use of molecular studies (involving whole genome sequencing) as a realistic approach to detect early emergence of resistant populations of parasites in areas where ACT is used. In select studies, low levels of $k 13$ mutations were detected in parasite isolates obtained from various regions of Africa [8, 24, 25]. In Nigeria, although chloroquine was replaced by artemisinin-based combinations as the drug of choice, a recent study observed that the percentage of children with malaria treated with un-recommended antimalarial monotherapy of chloroquine and sulfadoxine/ pyrimethamine was still significantly high at $11.6 \%$ and $10 \%$, respectively, compared to ACT at $7.5 \%$ [26]. As sometimes observed, heightened use of ACT and other anti-malarial therapies may confer transmission advantage to drug resistant $P$. falciparum. Consequently, selfmedicating and indiscriminate drug use may influence the circulating population of parasite strains, thus, selecting for mutant parasites through selective pressure. There is a need for continuous monitoring for the emergence of new anti-malarial-associated mutations that may arise in these high-endemic and highly populated areas, such as Nigeria.

This study, using Sanger and NGS methods, reported here, reveal the mutations identified in the Pfcrt, $P f m d r 1$ and $P f k 13$ genes and highlight the detection of rare mutations in the $P f m d r 1$ gene in Nigeria.

\section{Methods \\ Study sites}

The study participants were drawn from two sites in Lagos State; Ikorodu and Amukoko. Investigators from the University of Lagos have been conducting routine surveillance for malaria in Ikorodu, a semi urban area and Amukoko an urban slum in Lagos state. The two sites are hyper-endemic for $P$. falciparum, with generally high malaria transmission and a seasonal peak from July to November. Samples were collected between July 2015 and July 2016. The samples from Amukoko were collected at the St. Matthew Catholic Hospital. In Ikorodu, located approximately $20 \mathrm{~km}$ from Lagos, study participants were enrolled at primary health centres, Ijede and Agura. 


\section{Study samples}

A cross section sample of research participants who were patients, who reported to these health care facilities, with history of headache, fever (axillary temperature $\geq 37.5^{\circ} \mathrm{C}$ ) and other clinical symptoms of malaria in the previous $24 \mathrm{~h}$, was screened for malaria infection by both malaria rapid diagnostic test (RDT) (SD Bioline-Pf/ PAN) and microscopy. Recruitment was on a voluntary basis with informed consent obtained from all participating patients/caregivers after due counseling. The consent forms and all information about the study participants were kept confidential. A questionnaire was administered to each research participant to collect information such as demographic data, history of fever, other symptoms, and medication history, and a case-controlled design was adopted. Healthy individuals who are staff and students at the College of Medicine, University of Lagos that were without symptoms of acute illness during screening, had no evidence or history of chronic illness, and had no parasitaemia upon examination were also enrolled as controls. Venous blood collected into EDTA container from each research participant was used for malaria diagnosis and spotted on Whatman ${ }^{\circledR} \# 3$ filter paper for molecular studies.

Patients were treated with a combination of artemether and lumefantrine (AL [Coartem $\left.{ }^{\circledR}\right]$ ), as recommended by the WHO and the Nigeria National Malaria Control Programme (NMCP). Samples from subjects aged 2 to 73 years with uncomplicated $P$. falciparum mono-infection confirmed by microscopy (parasite density of 2000 to 199,999 asexual parasites/ $\mu \mathrm{L}$ ) were used for further study.

\section{Sanger sequencing}

Genomic DNA was isolated from three punched-out circles from a dried blood spot or $50 \mu$ l of whole blood taken at enrollment (day 0) using a QIAamp DNA minikit (Qiagen, Valencia, CA) according to the manufacturer's protocol.

Confirmation of $P$. falciparum infection and DNA quality assessment were conducted using photo-induced electron transfer (PET)-PCR [27]. The confirmed P. falciparum samples were used to amplify the $P f k 13$ propeller domain, $P f m d r 1$ and $P f c r t$ genes using previously described methods [28, 29]. Briefly, PCR was performed to amplify the full-length $P f c r t, P f m d r 1$ and $P f k 13$ genes using the New England BioLabs (NEB) High Fidelity PCR kit (New England BioLabs, USA; catalog no. 51104) according to the manufacturer's instructions with a $20 \mu \mathrm{L}$ master mix preparation using the $5 \mu \mathrm{L}$ GC buffer and 1-2 $\mu \mathrm{L}$ of total genomic DNA. PCR products were confirmed after ExoSAP cleanup using 1.5\% agarose gel electrophoresis. The samples were then genotyped by direct sequencing using an Applied Biosystems 3130 capillary sequencer and analysed with Geneious Pro R7 (Biomatters, Inc., Auckland, New Zealand) to identify specific SNPs. Sequencing and data analysis was performed at the Centers for Disease Control and Prevention, Atlanta, GA.

\section{Next Generation Sequencing (NGS)}

The recently developed MaRS protocol was employed as previously described [30]. Briefly, a Sequal Prep kit (ThermoFisher Scientific; catalog number A1051001) was used to purify and normalize all the PCR products to 1.0 to $2.0 \mathrm{ng} / \mu \mathrm{L}$ and $10.0 \mu \mathrm{L}$ of each $\mathrm{PCR}$ product was then pooled for each sample. Illumina-supported sequencing adaptors and unique sequence indices were added to the pooled PCR amplicons using the Illumina Nextera XT kit (Illumina; catalog numbers FC-131-1096 and FC-1311002) to generate a unique sequence barcode identifier (ID) for each sample and all pooled gene targets. Next, a limited-cycle PCR was performed to amplify the fragmented DNA and add unique (sequence) indices to each pooled sample. PCR products were then size selected $(<300 \mathrm{bp})$, normalized, and purified using Agent court AM pure XP beads (Beckman Coulter Genomics; catalog number A63881). Following normalization, $5.0 \mu \mathrm{L}$ of each library was pooled, allowing up to 384 libraries (or individual samples) to be pooled on a single Illumina MiSeq run. For data quality control of the FASTQ files obtained, only Q30 scores of $>98 \%$ were used. Low phred scores sequences were not considered in the analysis. The reference used to call the SNP and the haplotypes was the P. falciparum 3D7. The bioinformatics analysis of the data was performed using the Geneious R10 software package. The threshold used for SNP calling was set at allele frequency of $>10 \%$.

\section{Results}

Detection of Pfk13, Pfcrt, andPfmdr1 mutations by Sanger and NGS methods

Clinical samples from 1500 patients in Ikorodu and 730 in Amukoko study sites which were screened for malaria by rapid diagnostic test (RDT) and microscopy yielded 98 malaria-positive samples that were evaluated further by PET-PCR methods.

Thirty-seven samples from patients with self-reported anti-malarial medication use that were positive for $P$. falciparum were selected for molecular studies. Amplification and sequencing of the $P f k 13, P f c r t$, and $P f m d r 1$ genes was successfully performed in 26 samples that passed the quality threshold based on a minimum CT value of 40; the rest samples had low parasitaemia and amplification was not successful. In order to determine the efficiency 
of using the Sanger and NGS methods to detect mutations in the clinical isolates, both methods were used for sequencing of the selected genes. The Sanger method was limited to sequencing of partial fragments within the genes, however, the NGS method allowed for the sequencing of the entire genes. Of the common fragments sequenced by both methods, more mutations and haplotypes were detected by NGS than by Sanger. Furthermore, additional mutations and haplotypes were identified in the regions that could only be sequenced by the NGS method, thus, the NGS method has shown to be a more sensitive detection method for the screening of mutations in these three genes (Tables 1,2). These results indicate the preeminence of NGS for use in the tracking of the emergence and spread of mutant alleles.

\section{Prevalence of mutations detected in the Pfk13, Pfcrt, and Pfmdr1 genes}

Since more mutations were detected by NGS than by Sanger, it was necessary to investigate the prevalence of mutations in the each of the Pfcrt, Pfmdr1, and Pfk 13 genes because some specific mutations in those genes have been shown to have implications for anti-malarial resistance. Mutations were detected in $73.1 \%$ of studied samples in the $P f k 13$ gene. Investigations of the common regions of the $P f k 13$ gene sequenced by both methods showed that the NGS method detected a propeller mutation $(\mathrm{Q} 613 \mathrm{H})$, which was undetected by the Sanger method. An additional two non-propeller mutations (K189T, H136N) in the Pfk13 gene were detected in the additional genomic regions sequenced by NGS only; see Table 1 . When evaluating the genomic regions sequenced by both Sanger and NGS methods, the mutations detected in the Pfcrt gene, the M74I, N75E and K76T mutations were found in only 7 (26.9\%) of the samples by Sanger and 9 (34.6\%) of the samples by NGS. The CVIET and CVMET haplotypes detected in the regions sequenced by both methods were found in one additional sample by NGS than by Sanger. Furthermore, four additional mutations (A220S, Q271E, I356T, and R371I) and three additional mutant haplotypes (SEII, SETI, AEIR) were identified in additional regions of the Pfcrt gene sequenced by NGS only; see Tables 1,2 . This result revealed that approximately one-third of the samples analysed had one or more Pfcrt mutations, including a key mutation, K76T, which has been previously associated with resistance to the formerly used chloroquine and the current alternate first-line ACT partner drug amodiaquine.

Evaluation of the mutations detected in the common regions of the Pfmdr1 gene sequenced by Sanger and NGS methods, revealed that the Y184F mutation
Table 1 Distribution of mutant and wild type alleles in the samples sequenced by Sanger and NGS methods

\begin{tabular}{|c|c|c|c|c|c|}
\hline \multirow[t]{2}{*}{ Gene } & \multirow[t]{2}{*}{ Mutations } & \multicolumn{2}{|c|}{ Sanger number (\%) } & \multicolumn{2}{|c|}{ NGS number (\%) } \\
\hline & & $\begin{array}{l}\text { Mutant } \\
\text { allele }\end{array}$ & Wild type & $\begin{array}{l}\text { Mutant } \\
\text { allele }\end{array}$ & Wild type \\
\hline \multirow[t]{7}{*}{ Pfert } & M74! & $7(26.9 \%)$ & $19(73.1 \%)$ & $9(34.6 \%)$ & $17(65.4 \%)$ \\
\hline & $\mathrm{N} 75 \underline{E}$ & $7(26.9 \%)$ & $19(73.1 \%)$ & $9(34.6 \%)$ & $17(65.4 \%)$ \\
\hline & K76I & $7(26.9 \%)$ & $19(73.1 \%)$ & $9(34.6 \%)$ & $17(65.4 \%)$ \\
\hline & A220요 & NA & NA & $7(26.9 \%)$ & $19(73.1 \%)$ \\
\hline & $\mathrm{Q} 271 \underline{\mathrm{E}}$ & NA & NA & $6(23 \%)$ & $20(77 \%)$ \\
\hline & 1356I & NA & NA & $8(30.8 \%)$ & $18(69.2 \%)$ \\
\hline & R371! & NA & NA & $7(26.9 \%)$ & $19(73.1 \%)$ \\
\hline \multirow[t]{6}{*}{ Pfmdr1 } & N86Y & $2(7.7 \%)$ & $24(92.3 \%)$ & $2(7.7 \%)$ & $24(92.3 \%)$ \\
\hline & Y184E & $17(65.4 \%)$ & $9(34.6 \%)$ & $22(84.6 \%)$ & $4(15.4 \%)$ \\
\hline & N504K & NA & NA & $4(15.4 \%)$ & $22(84.6 \%)$ \\
\hline & N649믐 & NA & NA & $3(11.5 \%)$ & $23(88.5 \%)$ \\
\hline & F938Y & NA & NA & $3(11.5 \%)$ & $23(88.5 \%)$ \\
\hline & S967Nㅡㄴ & NA & NA & $1(3.8 \%)$ & $25(96.2 \%)$ \\
\hline \multirow[t]{3}{*}{ Pfk 13} & Q613브 & Nil & $100 \%$ & $1(3.8 \%)$ & $25(96.2 \%)$ \\
\hline & K189I & NA & NA & $17(65.4 \%)$ & $9(34.6 \%)$ \\
\hline & $\mathrm{H} 136 \mathrm{~N}$ & NA & NA & $1(3.8 \%)$ & $25(96.2 \%)$ \\
\hline
\end{tabular}

The mutations detected in the Pfcrt, Pfmdr1, and Pfk 13 genes that were sequenced by Sanger and NGS methods are displayed. The total number of samples that carried the specific mutation is shown and percent is given in parentheses. NA, not applicable. Unlike NGS, Sanger could only sequence fragments and not full length of the genes

and the NF haplotype were found in five additional samples by NGS compared to Sanger. In the regions sequenced by NGS only, an additional four mutations (N504K, N649D, S967N, F938Y) and 5 mutant hap-

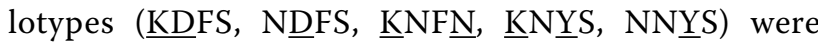
detected; see Tables 1, 2. The S967N, N649D, F938Y, and N504K mutations that were detected in 1, 3, 3 and 4 of sample population, respectively, are considered rare in light of the fact that they have not been previously reported in the study area. There was high prevalence of the Y184F as well as the NF haplotype, having been detected in $84.6 \%$ of clinical isolates analysed. Consistent with this finding, the NF haplotype has been previously identified as the most commonly reported haplotype in Africa [20].

\section{Evaluation of the number of mutations detected in clinical isolates from each patient sample}

Since there was a high preponderance of mutations found in the patient samples analysed, it was necessary to evaluate the number of mutations identified in each of the clinical isolates. Six of $26(23 \%)$ of the sequenced isolates carried mutations in one or more codon position in each of the Pfk13, Pfcrt and Pfmdr1 
Table 2 Distribution of the haplotypes identified in the samples sequenced by Sanger and NGS methods

\begin{tabular}{|c|c|c|c|}
\hline Gene & Haplotypes & Sanger (number \%) & NGS (number \%) \\
\hline \multirow[t]{7}{*}{ Pfcrt } & CVIET & 7 (26.9\%) & $8(30.8 \%)$ \\
\hline & CVMET & 0 & $1(3.8 \%)$ \\
\hline & CVMNK (wt) & $19(73.1 \%)$ & $17(65.4 \%)$ \\
\hline & SEll & ND & 1 (3.8\%) \\
\hline & $\underline{\text { SETI }}$ & ND & $6(23 \%)$ \\
\hline & AEEIR & ND & $1(3.8 \%)$ \\
\hline & AQIR & ND & $18(69.2 \%)$ \\
\hline \multirow[t]{9}{*}{ Pfmdr1 } & $\underline{\mathrm{YF}}$ & $2(7.7 \%)$ & $2(7.7 \%)$ \\
\hline & $\mathrm{NE}$ & $15(57.7 \%)$ & $22(84.6 \%)$ \\
\hline & NY (wt) & $9(34.6 \%)$ & $2(7.7 \%)$ \\
\hline & $\underline{K} N Y \underline{S}$ & ND & $1(3.8 \%)$ \\
\hline & $\underline{\text { KDFS }}$ & ND & $2(7.7 \%)$ \\
\hline & NDEFS & ND & $1(3.8 \%)$ \\
\hline & $\underline{\mathrm{KNF}} \underline{\mathrm{N}}$ & ND & $1(3.8 \%)$ \\
\hline & NNYYS & ND & $2(7.7 \%)$ \\
\hline & NNFS (wt) & ND & $19(73.1 \%)$ \\
\hline
\end{tabular}

The haplotypes identified in the Pfcrt and Pfmdr1 genes that were sequenced by Sanger and NGS methods are shown. Wt = wild type. The total number of samples that carried the specific haplotype is indicated and percent is given in parentheses. $\mathrm{ND}=$ not detected. The ability to detect certain haplotypes by Sanger is limited because only fragments and not full length gene could be sequenced by the method. The Pfcrt mutant haplotypes CVIET, CVMET and wild type CVMNK are based on protein sequence at codons $72-76$ while Pfcrt mutant haplotypes SEII, SETI, AEIR and wild type AQIR are based on protein sequence at codons $220,271,356$ and 371 . The mutant Pfmdr1 haplotypes $\underline{\text { F, NE }}$ and wild type NY are based on protein sequence at codons 86 and 184 while mutant

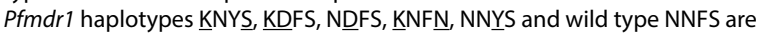
based on protein sequence at codons 504,649,938 and 967

genes. Nineteen of the $26(73.1 \%)$ isolates had one or more Pfk 13 mutations, 9 of the 26 (34.6\%) isolates had one or more Pfcrt mutations, and 22 of the 26 (84.6\%) isolates had one or more Pfmdr1 mutations; see Fig. 1. The high prevalence of Pfmdr1 mutations is largely attributed to the Y184F mutation, being found in $96 \%$ of the isolates that had mutations in this gene.

\section{Discussion}

To proactively undertake surveillance for mutations that may lead to future resistance to $\mathrm{ACT}$, sequencing data was generated from clinical isolates in individual patient samples in a high malaria endemic area using Sanger and NGS methods. Unlike other PCR based genotyping methods, which are only well suited for identification of major resistance alleles, the NGS method can accurately capture mixed infection haplotypes and detect minor resistance alleles as low as $1 \%[31,32]$. This study took advantage of the sensitivity of the NGS method in detecting the naturally circulating mutations in $P$. falciparum from individual patient samples. The results showed compelling evidence that the NGS method was more effective, detecting more mutations and haplotypes that were not identified by the Sanger method, thus, highlighting the efficacy of this method for surveillance of circulating anti-malarial resistance-associated alleles.

In $73.1 \%$ of the clinical isolates studied, Pfk 13 mutations consisting of two non-propeller mutations K189T and $\mathrm{H} 136 \mathrm{~N}$ and one propeller mutation $\mathrm{Q} 613 \mathrm{H}$ were detected. Previously reported data from samples in this study area of Lagos, Nigeria showed no $k 13$ propeller mutations in the 89 samples evaluated by Sanger method [24]. Though the frequency of the propeller Q613H allele was low and its association with any resistance phenotype is unknown, its presence confirms the possibility of independent development of $k 13$ resistance genotypes as has been reported from other sites in Africa [8, 24, and 25]. Previous data from Africa has reported low levels of various $k 13$ propeller mutations, which is consistent with the findings of this research [24,33]. There was a high prevalence $(65.4 \%)$ involving a K189T mutation which has been previously associated with anti-malarial resistance, however, it is noteworthy that multiple mutations are required to functionally confer drug resistance [34].

Following reduction in chloroquine efficacy in the treatment of malaria and in line with the WHO recommendation, ACT was adopted and deployed as the first line drug in the therapy of malaria by the Nigeria malaria control programme in 2005 [35]; however, studies conducted years after chloroquine was withdrawn continued to show mutations related to chloroquine-resistance phenotypes in Pfcrt and Pfmdr1 genes in parasite populations in Nigeria [36, 37]. Evidence abound that the occurrence of mutation is a continuous event, even after withdrawal of a drug. This is consistent with findings in Ethiopia which identified the Pfcrt K76T mutation, associated with chloroquine resistance, in $100 \%$ of their clinical isolates, even though chloroquine was withdrawn in Ethiopia in 1999 [38]. In contrast, significant decrease in Pfcrt mutations (K76T) associated with clinical resistance to chloroquine and increase in the wild type Pfcrt allele (K76) which correlated with reversal to chloroquine susceptibility has been reported in some African countries, many years after chloroquine withdrawal as a first-line treatment for malaria [39-41]. In addition, the K76T mutation identified in $34.6 \%$ of patient samples analysed has been previously associated with chloroquine and amodiaquine resistance and the triple mutant CVIET haplotype which was identified in $26.9 \%$ of the clinical isolates has been previously associated with chloroquine resistance [38]. The moderate prevalence of the K76T mutation and CVIET haplotype in this region is a significant finding which may suggest that selective pressure is still being evoked, possibly by previous indiscriminate chloroquine use, or occasional chloroquine use in the study area. Chloroquine use in indiscriminate 


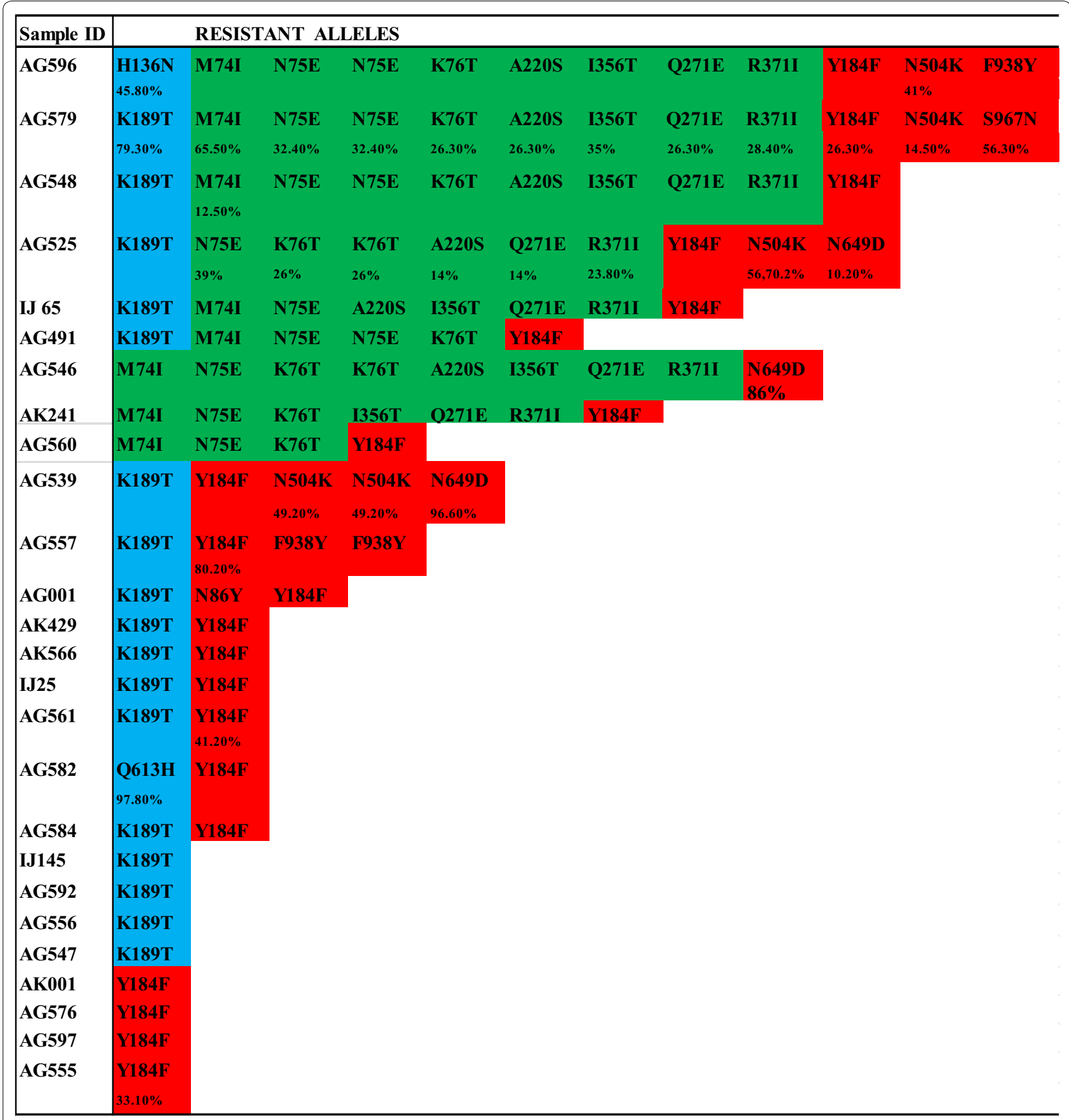

Fig. 1 The individual sample data indicating the mutations detected by NGS in the PfK13 (blue), Pfcrt (green), and Pfmdr1 (red) genes. Variant frequency (VF) is the minimum fraction of reads that must contain a base for it to be called a variation (i.e. polymorphism). VF values of less than $100 \%$ are displayed with the specific mutation

self-medication in the treatment of presumed malaria is still adopted, partly because the drug is widely available and accessible [26].

The need for laboratory-based diagnosis of malaria cannot be overemphasized, considering the vagueness of symptoms. For example, a recent study conducted in the study area reported over diagnosis and overtreatment of malaria, giving ACT to children that were later confirmed negative for malaria [42]. Furthermore, the association of $\mathrm{K} 76 \mathrm{~T}$ with amodiaquine resistance could have 
important implications for the effectiveness of the ACT (artesunate/amodiaquine) which is currently being used as the alternate first line treatment for malaria in high endemic Nigeria.

The variability of strains circulating in a high malariaendemic population, such as Nigeria, is not novel; however, it emphasizes the need for the molecular surveillance of these strains in the population, particularly those strains harbouring mutations with a previously demonstrated association with anti-malarial resistance.

The Pfmdr1 N86Y and Y184F mutations which are reported in the clinical isolates studied are not associated with resistance to lumefantrine, compared with previous reports on wild-type haplotypes $[8,20,43]$. It has been observed that the N86 wild-type allele is more prevalent in recurrent infections following treatment with artemether/lumefantrine [18, 44], and this was confirmed by a recent meta-analysis of 31 clinical trials that revealed a five-fold risk of parasite recrudescence in patients infected with parasites expressing the wild-type N86 following artemether/lumefantrine treatment compared with those infected with N86Y mutant parasites [19]. Furthermore, the N86Y mutation has been shown to increase susceptibility to lumefantrine and while promoting resistance to chloroquine and amodiaquine [20], thus, the low prevalence of the N86Y mutation $(7.7 \%)$ could imply that selective drug pressures are encouraging the preponderance of the wild-type phenotype to promote drug resistance. The effect of N86Y mutation on parasite response to chloroquine and amodiaquine is significant in parasites expressing the CVIET Pfcrt variant [20], thus, making the evaluation of regional associations between Pfmdr1 and Pfcrt haplotypes important. Information on the geographic distribution of Pfmdr1 and Pfcrt haplotypes and local selective drug pressures may help to inform decisions on selecting optimal ACT regimen.

High prevalence of the wild-type N86 allele (84.6\%) in this study samples, that have been linked to decrease susceptibility to artemether/lumefantrine [19] portend negative implication for its future use. Currently, the selective pressure on artemisinin in Nigeria is low because its usage is still recent and access and usage of it as a frontline ACT is still limited [2]. Although it is unclear whether the clinical isolates in this study are directly resistant to any anti-malarial drugs, the high preponderance of mutations makes the association to resistance reasonable, and circulation of these mutations has the potential to culminate in treatment failure and repeat visits to the clinic. The argument is that, the increased use of ACT and preponderance of molecular markers linked to decrease sensitivity to its partner drug will increase the selective pressure for artemisinin resistance. Strikingly, some of the individual isolates carry mutations that cut across 2 or 3 of the resistance-associated genes, possibly allowing for the manifestation of multi-drug resistant parasites. Mutations in the Pfk13, Pfcrt and Pfmdr1 genes were found in 6 of 26 (23\%) of the sequenced isolates while mutations involving $P f k 13$ and $P f m d r 1$ genes were exhibited in 9 of 26 (34.6\%) isolates, with amino acid substitutions in one or more codon in each of $P f c r t$ and $P f m d r 1$ genes in 2 of $26(7.7 \%)$ isolates.

Information on the preponderance of anti-malarial drug resistance-associated mutations is vital to understanding how resistance patterns emerge. As malaria control strategies intensify in the region and the country enters the pre-elimination phase, continued molecular surveillance using sensitive methods, such as the NGS, will be useful to track the evolution and prevalence of mutations in resistance associated genes. A study to correlate mutations detected with the clinical outcomes of treatment was not performed and this is a limitation to this study.

\section{Conclusion}

The findings of rare mutations (N504K, N649D, F938Y and $\mathrm{S} 967 \mathrm{~N}$ ) in genes associable with anti-malarial resistance in clinical isolates in this study, on the one hand, provide important insights into the continued evolving nature of the problems of malaria parasites when exposed to therapeutic interventions. On the other hand, in a country as Nigeria, with high endemicity of malaria, the emergence of mutant parasite may amount to a serious adverse public health consequence. Arguably, it is imperative that there is a valuable need for the adoption of sensitive molecular methods in malaria surveillance and control effort to ensure early detection of possible resistant strains to the highly recommended artemisininbased combination therapy.

\section{Acknowledgements}

We thank Dr. Eldin Talundzic, Dr. Naomi Lucchi, and Dr. Venkatachalam Udhayakumar from the Center for Disease Control and Prevention Atlanta, GA and Beth Pfotenhauer and Jennifer Lentz of City of Milwaukee Health Department Laboratory for their helpful suggestions and technical assistance. In addition, we thank the Fulbright Commission, Bureau of Cultural and Educational Exchange, State Department, USA, for the award of the Fulbright Visiting Research Grant to A. Idowu, the College of Health Sciences, University of Wisconsin- Milwaukee for hosting A. Idowu and providing financial assistance to the project, the City of Milwaukee Health Department Laboratory for internship placement, and the University of Lagos, Nigeria for financial assistance.

\section{Authors' contributions}

AOI, WAO, AA and UEM designed the study. AOI, WAO, AA, SB, and MK implemented the study. $\mathrm{AOl}$ and $\mathrm{AA}$ analysed the data. $\mathrm{AOI}$ and $\mathrm{AA}$ wrote the manuscript. AOI, AA, WAO, VVB, CB, Jl, and SB revised the manuscript. All authors read and approved the final manuscript.

\section{Funding}

This work was supported by funds from College of Health Sciences, University of Wisconsin-Milwaukee and the University of Lagos, Nigeria. The Fulbright Commission, Bureau of Cultural and Educational Exchange, State Department, 
USA, awarded Fulbright Visiting Research Grant (Grantee ID 15160886) to AOI. The Milwaukee Health Department Laboratory in Milwaukee WI and the Division of Parasitic Diseases and Malaria, Centers for Disease Control and Prevention, Atlanta, Georgia funded the aspects of studies on malaria gene polymorphism.

\section{Availability of data and materials}

The datasets used and/or analysed during the current study are available from the corresponding author on reasonable request

\section{Ethics approval and consent to participate}

The study was approved by the Ethics and Human Subjects Committee of College of Medicine, University of Lagos, Nigeria (Protocol Number CMUL/ HREC/05/17/205). Written informed consent and/or assent were obtained from study participants and/or their guardians. The collaborating investigators at Centers for Disease Control and Prevention (CDC) obtained permission from the CDC Human Subjects Office (CGH tracking \# 2017-100) to participate in the genotyping of the samples under anonymous conditions without access to any personal identifying information.

\section{Consent for publication}

Not applicable.

\section{Competing interests}

The authors declare that they have no competing interests.

\section{Author details}

1 Department of Biomedical Sciences, College of Health Sciences, University of Wisconsin, 2400 E. Hartford Avenue, Milwaukee, WI 53211, USA. ${ }^{2}$ Department of Pharmaceutics and Pharmaceutical Technology, Faculty of Pharmacy, University of Lagos, Lagos, Nigeria. ${ }^{3}$ City of Milwaukee Health Department Laboratory, Milwaukee, USA. ${ }^{4}$ ANDI Centre of Excellence in Malaria Diagnosis, College of Medicine, University of Lagos, Lagos, Nigeria. ${ }^{5}$ Molecular Pathogenesis Laboratory, National Center for Emerging and Zoonotic Infectious Diseases, Centers for Disease Control and Prevention, Atlanta, GA, USA.

${ }^{6}$ Department of Biology, North Life Science 317, San Diego State University, San Diego, CA 92182, USA.

\section{Received: 22 February 2019 Accepted: 6 September 2019} Published online: 18 September 2019

\section{References}

1. WHO. Global Malaria. Programme World Malaria Report 2014. Geneva: World Health Organization; 2014.

2. National Malaria Elimination Program (NMEP, Commission (NPopC) NP, National Bureau of Statistics (NBS, ICF International. Nigeria Malaria Indicator Survey 2015. 2016.

3. WHO. World malaria report 2016. Geneva: World Health Organization; 2016.

4. WHO. Guidelines for the treatment of malaria. Geneva: World Health Organization; 2015.

5. Sowunmi A, Akano K, Ntadom G, Ayede Al, Ibironke FO, Aderoyeje T, et al. Therapeutic efficacy and effects of artemisinin-based combination treatments on uncomplicated Plasmodium falciparum malaria -associated anaemia in Nigerian children during seven years of adoption as first-line treatments. Infect Dis Poverty. 2017;6:36.

6. Dondorp AM, Nosten F, Yi P, Das D, Phyo AP, Tarning J, et al. Artemisinin resistance in Plasmodium falciparum malaria. N Engl J Med. 2009;361:455-67.

7. Ariey F, Witkowski B, Amaratunga C, Beghain J, Langlois A-C, Khim N, et al. A molecular marker of artemisinin-resistant Plasmodium falciparum malaria. Nature. 2014;505:50-5.

8. Ashley EA, Dhorda M, Fairhurst RM, Amaratunga C, Lim P, Suon S, et al. Spread of artemisinin resistance in Plasmodium falciparum malaria. N Engl J Med. 2014;371:411-23.

9. Miotto O, Amato R, Ashley EA, MacInnis B, Almagro-Garcia J, Amaratunga $C$, et al. Genetic architecture of artemisinin-resistant Plasmodium falciparum. Nat Genet. 2015;47:226-34.

10. WHO. Minutes of the Expert Review Committee on K13 molecular marker of artemisinin resistance. Geneva: World Health Organization; 2014.
11. WHO. Artemisinin and artemisinin-based combination therapy resistance: status report. Geneva: World Health Organization; 2016. http://apps.who. int/iris/handle/10665/208820.

12. Ecker A, Lehane AM, Clain J, Fidock DA. PfCRT and its role in antimalarial drug resistance. Trends Parasitol. 2012;28:504-14.

13. Djimdé A, Doumbo OK, Cortese JF, Kayentao K, Doumbo S, Diourté Y, et al. A molecular marker for chloroquine-resistant falciparum malaria. N Engl J Med. 2001;344:257-63.

14. Reed MB, Saliba KJ, Caruana SR, Kirk K, Cowman AF. Pgh1 modulates sensitivity and resistance to multiple antimalarials in Plasmodium falciparum. Nature. 2000;403:906-9.

15. Lekostaj JK, Amoah LE, Roepe PD. A single S1034C mutation confers altered drug sensitivity to PFMDR1 ATPase activity that is characteristic of the 7G8 isoform. Mol Biochem Parasitol. 2008;157:107-11.

16. Sanchez CP, Rotmann A, Stein WD, Lanzer M. Polymorphisms within PfMDR1 alter the substrate specificity for anti-malarial drugs in Plasmodium falciparum. Mol Microbiol. 2008;70:786-98.

17. Conrad MD, LeClair N, Arinaitwe E, Wanzira H, Kakuru A, Bigira V, et al. Comparative impacts over 5 years of artemisinin-based combination therapies on Plasmodium falciparum polymorphisms that modulate drug sensitivity in Ugandan children. J Infect Dis. 2014;210:344-53.

18. Malmberg M, Ferreira PE, Tarning J, Ursing J, Ngasala B, Björkman A, et al. Plasmodium falciparum drug resistance phenotype as assessed by patient antimalarial drug levels and its association with pfmdr1 polymorphisms. $J$ Infect Dis. 2013;207:842-7.

19. Venkatesan M, Gadalla NB, Stepniewska K, Dahal P, Nsanzabana C, Moriera C, et al. Polymorphisms in Plasmodium falciparum chloroquine resistance transporter and multidrug resistance 1 genes: parasite risk factors that affect treatment outcomes for P. falciparum malaria after artemether-lumefantrine and artesunate-amodiaquine. Am J Trop Med Hyg. 2014;91:833-43.

20. Veiga MI, Dhingra SK, Henrich PP, Straimer J, Gnädig N, Uhlemann A-C, et al. Globally prevalent PfMDR1 mutations modulate Plasmodium falciparum susceptibility to artemisinin-based combination therapies. Nat Commun. 2016:7:11553.

21. Amaratunga C, Lim P, Suon S, Sreng S, Mao S, Sopha C, et al. Dihydroartemisinin-piperaquine resistance in Plasmodium falciparum malaria in Cambodia: a multisite prospective cohort study. Lancet Infect Dis. 2016;16:357-65.

22. Phuc BQ, Rasmussen C, Duong TT, Dong LT, Loi MA, Ménard D, et al. Treatment failure of dihydroartemisinin/piperaquine for Plasmodium falciparum malaria, Vietnam. Emerg Infect Dis. 2017:23:715-7.

23. Witkowski B, Duru V, Khim N, Ross LS, Saintpierre B, Beghain J, et al. A surrogate marker of piperaquine-resistant Plasmodium falciparum malaria: a phenotype-genotype association study. Lancet Infect Dis. 2017:17:174-83.

24. Kamau E, Campino S, Amenga-Etego L, Drury E, Ishengoma D, Johnson $\mathrm{K}$, et al. K13-propeller polymorphisms in Plasmodium falciparum parasites from sub-Saharan Africa. J Infect Dis. 2015;211:1352-5.

25. Talundzic E, Ndiaye YD, Deme AB, Olsen C, Patel DS, Biliya S, et al. Molecular epidemiology of Plasmodium falciparum kelch13mutations in Senegal determined by using targeted amplicon deep sequencing. Antimicrob Agents Chemother. 2017:61:2116.

26. National Population Commission, Abuja. Nigeria Demographic Health Survey. 2013

27. Lucchi NW, Narayanan J, Karell MA, Xayavong M, Kariuki S, DaSilva AJ, et al. Molecular diagnosis of malaria by photo-induced electron transfer fluorogenic primers: PET-PCR. PLOS ONE. 2013;8:e56677.

28. Talundzic E, Chenet SM, Goldman IF, Patel DS, Nelson JA, Plucinski MM, et al. Genetic analysis and species specific amplification of the artemisinin resistance-associated kelch propeller domain in $P$. falciparum and $P$. vivax. PLoS ONE. 2015;10:e0136099.

29. Griffing S, Syphard L, Sridaran S, McCollum AM, Mixson-Hayden T, Vinayak $\mathrm{S}$, et al. pfmdr1 amplification and fixation of pfcrt chloroquine resistance alleles in Plasmodium falciparum in Venezuela. Antimicrob Agents Chemother. 2010;54:1572-9.

30. Talundzic E, Ravishankar S, Kelley J, Patel D, Plucinski M, Schmedes S, et al. Next-Generation sequencing and bioinformatics protocol for malaria drug resistance marker surveillance. Antimicrob Agents Chemother. 2018;62:e02474. 
31. Flaherty P, Natsoulis G, Muralidharan O, Winters M, Buenrostro J, Bell J, et al. Ultrasensitive detection of rare mutations using next-generation targeted resequencing. Nucleic Acids Res. 2012;40:e2.

32. Pulido-Tamayo S, Sánchez-Rodríguez A, Swings T, Van den Bergh B, Dubey A, Steenackers $\mathrm{H}$, et al. Frequency-based haplotype reconstruction from deep sequencing data of bacterial populations. Nucleic Acids Res. 2015:43:105.

33. Takala-Harrison S, Laufer MK. Antimalarial drug resistance in Africa: key lessons for the future. Ann N Y Acad Sci. 2015;1342:62-7.

34. Boussaroque A, Fall B, Madamet M, Camara C, Benoit N, Fall M, et al. Emergence of mutations in the K13propeller gene of Plasmodium falciparum Isolates from Dakar, Senegal, in 2013-2014. Antimicrob Agents Chemother. 2016;60:624-7.

35. Federal Ministry of Health, Abuja. Nigeria national malaria treatment policy. 2005.

36. Oladipo OO, Wellington OA, Sutherland CJ. Persistence of chloroquineresistant haplotypes of Plasmodium falciparum in children with uncomplicated malaria in Lagos, Nigeria, four years after change of chloroquine as first-line antimalarial medicine. Diagn Pathol. 2015;10:41.

37. Oyebola KM, Idowu ET, Olukosi YA, Awolola TS, Amambua-Ngwa A. Pooled-DNA sequencing identifies genomic regions of selection in Nigerian isolates of Plasmodium falciparum. Parasit Vectors. 2017;10:320.

38. Golassa L, Enweji N, Erko B, Aseffa A, Swedberg G. High prevalence of pfcrt-CVIET haplotype in isolates from asymptomatic and symptomatic patients in south-central Oromia, Ethiopia. Malar J. 2014;13:120.

39. Frosch AEP, Laufer MK, Mathanga DP, Takala-Harrison S, Skarbinski J, Claassen CW, et al. return of widespread chloroquine-Sensitive Plasmodium falciparum to Malawi. J Infect Dis. 2014;210:1110-4.
40. Mwanza S, Joshi S, Nambozi M, Chileshe J, Malunga P, Kabuya BB, et al. The return of chloroquine-susceptible Plasmodium falciparum malaria in Zambia. Malar J. 2016;15:584.

41. Mekonnen SK, Aseffa A, Berhe N, Teklehaymanot T, Clouse RM, Gebru T, et al. Return of chloroquine-sensitive Plasmodium falciparum parasites and emergence of chloroquine-resistant Plasmodium vivax in Ethiopia. Malar J. 2014;13:244.

42. Oladosu OO, Oyibo WA. Overdiagnosis and Overtreatment of malaria in children that presented with fever in Lagos, Nigeria. International Scholarly Research Notices. 2013. https://www.hindawi.com/journals/ isrn/2013/914675/.

43. Happi CT, Gbotosho GO, Folarin OA, Sowunmi A, Hudson T, O'Neil M, et al. Selection of Plasmodium falciparum multidrug resistance gene 1 alleles in asexual stages and gametocytes by artemether-lumefantrine in Nigerian children with uncomplicated falciparum malaria. Antimicrob Agents Chemother. 2009;53:888-95.

44. Sisowath C, Strömberg J, Martensson A, Msellem M, Obondo C, Björkman $\mathrm{A}$, et al. In vivo selection of "Plasmodium falciparum pfmdrl" $86 \mathrm{~N}$ coding alleles by artemether-lumefantrine (Coartem). J Infect Dis. 2005;191:1014-7.

\section{Publisher's Note}

Springer Nature remains neutral with regard to jurisdictional claims in published maps and institutional affiliations.
Ready to submit your research? Choose BMC and benefit from:

- fast, convenient online submission

- thorough peer review by experienced researchers in your field

- rapid publication on acceptance

- support for research data, including large and complex data types

- gold Open Access which fosters wider collaboration and increased citations

- maximum visibility for your research: over $100 \mathrm{M}$ website views per year

At BMC, research is always in progress.

Learn more biomedcentral.com/submissions 